\title{
The relationship between trauma and beliefs about hearing voices: a study of psychiatric and non-psychiatric voice hearers
}

\author{
E. M. Andrew ${ }^{1,2 *}$, N. S. Gray ${ }^{2,3}$ and R. J. Snowden ${ }^{2}$ \\ ${ }^{1}$ Psychology Department, Royal Glamorgan Hospital, Llantrisant, UK \\ ${ }^{2}$ School of Psychology, Cardiff University, Cardiff, UK \\ ${ }^{3}$ South Wales Forensic Psychiatric Service, Caswell Clinic, Glanrhyd Hospital, Bridgend, UK
}

Background. Cognitive models suggest that distress associated with auditory hallucinations is best understood in terms of beliefs about voices. What is less clear is what factors govern such beliefs. This study aimed to explore the way in which traumatic life events contribute towards beliefs about voices and any associated distress.

Method. The difference in the nature and prevalence of traumatic life events and associated psychological sequelae was compared in two groups of voice hearers: psychiatric voice hearers with predominantly negative beliefs about voices $(\mathrm{PVH})$ and non-psychiatric voice hearers with predominantly positive beliefs about voices (NPVH). The data from the two groups were then combined in order to examine which factors could significantly account for the variance in beliefs about voices and therefore levels of distress.

Results. Both groups reported a high prevalence of traumatic life events although significantly more PVH reported trauma symptoms sufficient for a diagnosis of post-traumatic stress disorder (PTSD). Furthermore, significantly more $\mathrm{PVH}$ reported experiencing childhood sexual abuse. Current trauma symptoms (re-experiencing, avoidance and hyperarousal) were found to be a significant predictor of beliefs about voices. Trauma variables accounted for a significant proportion of the variance in anxiety and depression.

Conclusions. The results suggest that beliefs about voices may be at least partially understood in the context of traumatic life events.

Received 15 May 2007; Revised 26 October 2007; Accepted 14 November 2007; First published online 4 January 2008

Key words: Auditory hallucinations, trauma.

\section{Introduction}

The cognitive-behavioural model of voice hearing posits that it is not the occurrence of voices per se that causes distress, rather it is beliefs about voices that elicit emotional and behavioural consequences (Chadwick \& Birchwood, 1994).

\section{Life events and beliefs about voices}

Chadwick \& Birchwood (1994) have argued that whether a voice is construed as malevolent or benevolent, powerful or benign, is influenced by core cognitive schemata that are likely to be autobiographical in nature, in that they are believed to be related to the individual's past and current life experiences and interpersonal relationships. It has been shown on

\footnotetext{
* Address for correspondence: Dr E. M. Andrew, School of Psychology, Cardiff University, Park Place, Cardiff CF10 3AT, Wales, UK.

(Email: AndrewL1@cf.ac.uk)
}

numerous occasions that the content of, and beliefs about, voices is associated with an individual's life history (Romme \& Escher, 1989, 1993; Bentall, 1990; Chadwick \& Birchwood, 1994) and the way that they feel about themselves (Close \& Garety, 1998). It could therefore be hypothesized that life events, particularly traumatic life events, could represent a mediating or contributory factor in the development of beliefs about voices. Indeed, Birchwood et al. (2000) describe how early life events and relationships may lead to a sense of subordination, and that those who experience such events are more likely to perceive themselves as being unable to control the voices and therefore perceive their voices as being more powerful. Morrison (2001) and Garety et al. (2001) implicate traumatic life events as one of the factors influencing the way in which anomalous, intrusive experiences such as hearing voices are interpreted. Despite trauma being implicated in this way, few studies have examined the incidence and impact of trauma (in terms of posttraumatic stress symptomatology) specifically within a 
voice-hearing population and there currently exists very little specific evidence supporting a relationship between trauma and beliefs about voices.

\section{Traumatic life events and voices}

A host of studies have detected a significantly higher prevalence of traumatic life experiences and associated psychological sequelae (i.e. symptoms of post-traumatic stress disorder, PTSD) in individuals with psychosis than in the general population (Mueser et al. 1998; Frame \& Morrison, 2001; Neria et al. 2002; Bebbington et al. 2004). Fewer studies have mapped the relationship between trauma and specific symptoms. Those that do exist tend to focus on the relationship between childhood abuse and the symptoms of psychosis.

Many have highlighted the correlation between childhood abuse and the experience of hearing voices (Ensink, 1992; Falmularo et al. 1992; Ross \& Joshi, 1992). Kilcommons \& Morrison (2005) found a significant relationship between the incidence of traumatic life events and the experience of hearing voices. In particular, they detected a strong relationship between lifetime incidence of sexual abuse and hearing voices. Read et al. (2003) detected a significant relationship between auditory hallucinations and abuse, in both childhood and adulthood, in a sample of over 200 individuals attending a community mental health centre. They also noted that those who had been abused were significantly more likely to experience command hallucinations to harm or kill themselves than the group that did not report abuse. Unfortunately, Read and colleagues obtained their data from medical notes and did not have the opportunity to interview any of the participants. The authors themselves note that previous research suggests that many abuse cases are unidentified by clinicians (Young et al. 2001; Lothian \& Read, 2002), and also that individuals with a diagnosis of 'schizophrenia' are especially unlikely to be asked about abuse (Read \& Fraser, 1998a). It is therefore likely that the design of their study may have led to an underestimation of the 'true' prevalence of abuse that, in turn, could have minimized the apparent relationship between abuse and hearing voices.

Of course, 'trauma' should not be limited in definition to sexual or physical abuse. Previous research indicates a high prevalence of other traumatic life events, including the death or murder of a close friend or relative, witnessing a natural or man-made disaster, and being involved in an accident (see Mueser et al. 1998). It is therefore important that studies examining the relationship between trauma and symptomatology do not focus exclusively on abuse.
The relationship between traumatic life events and beliefs about voices

The current study was designed to examine the relationship between trauma, and in particular current symptoms relating to trauma, and beliefs about voices by involving two groups of voice hearers: psychiatric voice hearers $(\mathrm{PVH})$ and non-psychiatric voice hearers (NPVH). The use of an NPVH group is a novel feature of our study. In most studies in this domain only PVH are used. By including a non-psychiatric population it was hoped that we would find a group of individuals who, perhaps by definition, do not experience significant distress associated with voices. According to the cognitive model, it was hypothesized that such individuals would hold more 'innocuous' or benevolent beliefs about their voices (e.g. Romme \& Escher, 1989). Indeed, previous research has suggested that there are individuals in the general population who hear voices but who 'cope' with the experience and do not require input from psychiatric services. Such individuals have reported more positive beliefs about the voices they hear (Romme \& Escher, 1989). In the current study we targeted such a group of individuals in order to explore the extent to which trauma and any persistent psychological sequelae associated with trauma (i.e. intrusions, avoidance and hyperarousal) could account for the differences between the groups in terms of beliefs about voices and associated distress.

A study conducted by Honig et al. (1998) suggests that NPVH may also report a history of traumatic life events. However, the authors discussed the presence of child sexual abuse only and, furthermore, did not describe the extent to which the trauma continued to affect the individuals in terms of current symptoms of intrusions, avoidance and hyperarousal. It is conceivable that such factors could play a role in the development and maintenance of beliefs about voices (see Morrison, 2001). A number of studies have noted ways in which trauma may be related to voices suggesting that traumatic life events may be associated with the onset of hearing voices (Romme \& Escher 1989 , 1993) or may influence the content of voice activity (Close \& Garety, 1998; Raune et al. 1999). Although studies have indicated the potential relationship between trauma and voices (e.g. Kilcommons \& Morrison, 2005), no study to our knowledge has examined the way in which trauma may be related specifically to beliefs about voices and ultimately the level of distress associated with voices, in particular by including a non-psychiatric sample.

The main aim of this study was therefore to compare the prevalence of trauma and associated trauma symptoms (re-experiencing, avoidance and hyperarousal) in two groups of voice hearers. The second 
aim was to combine the groups to determine the extent to which trauma variables could account for the variance in beliefs about voices. It was hoped that this would help to further elucidate the way in which trauma contributes towards the distress associated with voices.

\section{Method}

\section{Participants}

All participants in the two groups included in this study, PVH and NPVH, were aged between 18 and 70, reported hearing voices in the previous month, and were willing and able to provide informed consent. Individuals were excluded from the project if they presented with an organic condition of which auditory hallucinations can be a symptom.

\section{PVH}

In addition to the criteria outlined above, PVH were excluded from this group if they were acutely distressed (to the extent where they would be unable participate or where participating would significantly exacerbate their mental state) either at the point of referral or at interview.

PVH were recruited from mental health services across four health authorities in South Wales: Gwent, Bro Taf, Iechyd Morganwwg and Dyfed Powys. Initially, 25 individuals who were in contact with psychiatric services were referred for participation in the study. Following referral, one individual declined to participate. Another participant was deemed, by the researcher, not to be able to provide informed consent. Both of these participants were male. A further participant was unable to complete all of the questionnaires because of her mental state at the time of interview. Her data are not included in the analysis.

\section{$N P V H$}

In terms of the NPVH, previous research suggests that there are certain identifiable groups of people who have elevated scores on 'positive symptom measures' such as hearing voices. These groups include those who believe in the paranormal (Thalbourne, 1994), those who have out-of-body experiences (McGreery \& Claridge, 1995), and members of new religious movements (Day \& Peters, 1999). In Western culture, people who report having these beliefs and experiences are often referred to as mediums or psychics (Tobert, 2001). It was therefore decided to target this group of individuals.

Several process issues had to be considered in the recruitment of this group of individuals. It has been noted in previous studies that NPVH are notoriously difficult to recruit. Understandably, they are reluctant to discuss their experiences with mental health professionals for fear of being pathologized. To facilitate recruitment, initial contacts were developed with mediums registered with the National Spiritualists Union and leaders of spiritualist churches in the South Wales area. Early conversations (not related to the research question) helped the principle researcher to understand alternative explanations of these experiences and to adopt the terminology used by this particular population. For example, mediums may report a number of 'unusual' experiences including seeing people or objects not visible to others (clairvoyance); sensing the characteristics associated with a communicating spirit (clairsentience); and hearing the voices of spirits (clairaudience). The current study focused specifically on the experience of clairaudience or 'hearing voices'. These terms were used with all mediums to demonstrate the researcher's awareness of an alternative, less pathologizing frame of reference. These initial conversations also helped the researcher to develop a non-judgemental and validating approach to interviewing mediums and psychics.

Several individuals who were initially contacted expressed an interest in the study and volunteered to participate. The 'snowball technique' was used thereafter. That is, individuals were given posters and information leaflets to pass on to other mediums they thought might be interested in the study. Individuals were excluded from the NPVH group if they reported current or past contact with psychiatric services. As this study aimed to look at what predicted the distress associated with voices rather than whether voices are a pathological phenomenon, meeting criteria for a psychiatric diagnosis was not an exclusion criteria and was therefore not assessed formally.

\section{Materials}

\section{Assessment of voices}

Psychotic Symptom Rating Scales - Auditory Hallucinations Subscale (PSYRATS-AH; Haddock et al. 1999). The PSYRATS-AH is an 11-item scale. The item pool taps the general symptom indices of frequency, duration, severity and intensity of distress and also symptomspecific dimensions of controllability, volume, location, negative content, beliefs about origin of voice, and disruption caused by the voice. Individuals are required to rate each item on a five-point ordinal scale ranging from 0 to 4 .

Belief About Voices Questionnaire-Revised (BAVQ-R; Chadwick et al. 2000). The BAVQ-R is a 35-item measure of people's beliefs about auditory hallucinations and 
their emotional and behavioural reactions to them. There are three subscales relating to beliefs about malevolence, benevolence and omnipotence. Two further subscales, 'resistance' and 'engagement', measure emotional and behavioural reactions. All responses are rated on a four-point ordinal scale measuring the extent to which the individual agrees with the statement.

\section{Trauma measures}

Post-traumatic Diagnostic Scale (PDS; Foa, 1995). The PDS provided the means of establishing the occurrence of traumatic life events. The PDS is a 49 -item, four-part self-report inventory that covers all main forms of traumatic life events. Individuals are asked to indicate (by ticking a box) which traumatic life events they had experienced in childhood (up to 16 years of age) and adulthood. The PDS is particularly useful as an aid to diagnosing PTSD (although it cannot be used in isolation to provide a diagnosis) as it taps all the main components listed in the DSM-IV (APA, 1994) criteria for PTSD. Although this measure has not been used in previous studies of individuals with psychosis, it was chosen for use in this study because it allowed research participants to report trauma in a less intrusive way than reporting it in interview format.

Impact of Events Scale (IES; Horowitz et al. 1979). The IES comprises 15 items that yield two subscales: intrusion and avoidance. Together the scales produce a total IES score that can serve as a useful indicator of the extent to which a traumatic event is reverberating in the mind. Items are scored on a four-point scale ranging from 'not at all' to 'often' to produce the two subscale scores and a total IES score.

\section{Measures of affect}

Beck Anxiety Inventory (BAI; Beck et al. 1988). The BAI is a 21-item self-report scale designed to measure the severity of anxiety symptoms. The individual is required to rate the severity of each symptom on a four-point scale ranging from 'not-at-all' (0) to 'severe' (3). Scores range from 0 to 63 , where a higher score represents greater symptom severity.

Beck Depression Inventory-II (BDI-II; Beck et al. 1996). The BDI-II is a 21-item self-report scale designed to assess the affective, cognitive, motivational, psychomotor and vegetative components of depression. Individuals are required to complete items in terms of the way they have felt in the previous 7 days.
Possible scores range from 0 to 63 , where a higher score indicates a greater severity of depression.

\section{Procedure}

All participants were required to provide written informed consent before completing the measures described above. All participants were interviewed by one author (E.M.A.). If no traumatic events were indicated in part one of the PDS (including in the 'other' category), the remainder of the questionnaire became redundant and was therefore not completed. In the event of no history of trauma, the IES was also omitted.

\section{Results}

The PVH group consisted of 22 individuals (13 males and nine females) aged between 21 and 70 years $($ mean $=39.55$, s.D. $=12.3)$. The mean length of time hearing voices was 12.91 years (S.D. $=10.10)$. The NVPH group consisted of 21 individuals (six males and 15 females) aged between 24 and 71 years $($ mean $=50.67$, s.D. $=11.3)$. Of the final sample, 19 described themselves as mediums and two as 'celtic seers'. The mean length of time hearing voices among the group was 30.62 years (s.D. $=15.77$ ). There were no significant differences between the groups on educational level. There were, however, significantly more $(p<0.05)$ males in the PVH group. The impact of this finding on the results was addressed and is presented later in this section. There was also a significant difference in the ages of the two groups $(p<0.05)$. We examined the effect of age on our outcome variables (e.g. length of time hearing voices, beliefs about voices, degree of distress, disruption associated with voices). As there was no significant relationship between age and any of these variables, we did not attempt to correct for it statistically.

Our key hypothesis was that the participants' experience of trauma would mediate their beliefs about voices. To explore this hypothesis, a series of linear multiple regression analyses were conducted examining the relationship between trauma variables and pertinent voice variables. To determine which variables to enter into the regression, we first examined which variables differed significantly between the groups. Table 1 shows that the PVH group exhibited the expected pattern of results. Compared to the NPVH group, they typically heard voices more frequently and for longer durations, the voices had more negative content, and they had less control and more distress from the voices. The PVH group believed the voices to be significantly more malevolent and omnipotent and reported significantly more resistant 
Table 1. Whole group comparison of voices, affect and occurrence of trauma

\begin{tabular}{|c|c|c|c|c|c|}
\hline Area of interest & Variable & & $\begin{array}{l}\text { PVH } \\
(n=22)\end{array}$ & $\begin{array}{l}\text { NPVH } \\
(n=21)\end{array}$ & $p$ value \\
\hline \multirow[t]{16}{*}{ Voice characteristics } & \multirow[t]{2}{*}{ Frequency } & Once a week & 2 & 11 & \multirow[t]{2}{*}{$<0.05$} \\
\hline & & Daily & 20 & 10 & \\
\hline & \multirow[t]{2}{*}{ Duration } & Seconds/minutes & 7 & 15 & \multirow[t]{2}{*}{$<0.05$} \\
\hline & & Hours & 15 & 6 & \\
\hline & \multirow[t]{2}{*}{ Volume } & Quieter/same & 14 & 20 & \multirow[t]{2}{*}{$<0.05$} \\
\hline & & Louder & 8 & 1 & \\
\hline & Negative & Rarely & 3 & 21 & $<0.001$ \\
\hline & Content & Majority/all & 19 & 0 & \\
\hline & \multirow[t]{2}{*}{ Distress } & Never/occasionally & 4 & 21 & \multirow{2}{*}{$<0.001$} \\
\hline & & Majority & 18 & 0 & \\
\hline & \multirow{2}{*}{$\begin{array}{c}\text { Degree of } \\
\text { distress }\end{array}$} & Mild & 2 & 21 & \multirow[t]{2}{*}{$<0.001$} \\
\hline & & Moderate-severe & 20 & 0 & \\
\hline & \multirow[t]{2}{*}{ Control } & Majority/always & 2 & 21 & \multirow[t]{2}{*}{$<0.001$} \\
\hline & & Rarely/never & 20 & 0 & \\
\hline & \multirow[t]{2}{*}{ Location } & Internal & 13 & 14 & \multirow[t]{2}{*}{ N.s. } \\
\hline & & External & 9 & 7 & \\
\hline \multirow[t]{5}{*}{ Beliefs about voices } & \multicolumn{2}{|l|}{ Malevolence } & $10.77(5.24)$ & $0.10(0.44)$ & $<0.001$ \\
\hline & \multicolumn{2}{|l|}{ Benevolence } & $3.09(5.3)$ & $12.38(4.4)$ & $<0.001$ \\
\hline & \multicolumn{2}{|l|}{ Omnipotence } & $13.00(3.75)$ & $3.33(2.1)$ & $<0.001$ \\
\hline & \multicolumn{2}{|l|}{ Resistance } & $17.86(8.39)$ & $0.62(1.66)$ & $<0.001$ \\
\hline & \multicolumn{2}{|l|}{ Engagement } & $7.09(8.71)$ & $15.62(5.34)$ & $<0.001$ \\
\hline \multirow[t]{2}{*}{ Affect } & \multirow{2}{*}{\multicolumn{2}{|c|}{$\begin{array}{l}\text { Anxiety } \\
\text { Depression }\end{array}$}} & $21.45(9.26)$ & $3.48(4.34)$ & $<0.001$ \\
\hline & & & $27(15.68)$ & $5.9(6.11)$ & $<0.001$ \\
\hline \multirow[t]{4}{*}{ No. of types of trauma } & \multicolumn{2}{|l|}{ Childhood } & 1.41 & 0.71 & N.s. \\
\hline & \multicolumn{2}{|l|}{ Adulthood } & 2.09 & 1.14 & N.s. \\
\hline & \multicolumn{2}{|l|}{ Lifespan } & 3.14 & 1.81 & $<0.05$ \\
\hline & \multicolumn{2}{|l|}{$\begin{array}{c}\text { No. reporting } \\
\text { sexual abuse }\end{array}$} & 11 & 3 & $<0.006$ \\
\hline
\end{tabular}

PVH, Psychiatric voice hearers; NPVH, non-psychiatric voice hearers; N.s., not significant.

Values in parentheses are standard deviations.

coping behaviours whereas the NPVH group believed their voices to be more benevolent and engaged more with the voices. The PVH group showed more current symptoms of depression and anxiety. Table 2 displays the data pertaining to trauma variables. Surprisingly, there were no significant group differences in the number of people who had a past traumatic event. However, it is worth noting that the percentage of participants who had had a traumatic event was high in both groups $(>75 \%)$. Overall, the PVH group had experienced a greater number of types of traumatic events over their lifetime, and the often found result of greater childhood sexual abuse in the PVH group was replicated. In examining the psychological sequelae of traumatic life events, only participants who reported at least one traumatic event were included in the analysis (PVH $n=18$, NPVH $n=16$ ). The PVH group had significantly greater symptomatology for every measure used, resulting in a greater rate of DSM-IV diagnosis of PTSD for the PVH group.
As noted earlier, there was an imbalance in gender between the groups. We therefore also compared $\mathrm{PVH}$ and NPVH groups split by gender. All group differences remained statistically significant for both genders, hence we only present the combined data here.

The regression analysis also excluded variables with high multi-collinearity (e.g. re-experiencing on the PDS and intrusions on the IES were highly correlated; $r>0.7)$ and therefore could be considered to be collinear (Bryman \& Cramer, 1997). In such an instance a variable was chosen that met the above criteria and was felt to reflect the construct in question (severity of trauma symptoms).

The following trauma variables were selected as independent variables: number of traumatic events, history of sexual assault in childhood, and the total score from the IES. Separate analyses were conducted for each of the dependent variables of beliefs about voices (malevolence, benevolence and omnipotence). 
Table 2. Comparison of specific trauma symptom variables

\begin{tabular}{clccc}
\hline & Variable & $n=18^{\mathrm{a}}$ & $n=16^{\mathrm{a}}$ & $p$ value \\
\hline Current trauma & No. re-experiencing & $3.11(1.41)$ & $1.50(1.51)$ & 0.005 \\
symptoms PDS & No. avoidance & $5.22(1.67)$ & $1.63(1.93)$ & 0.001 \\
& No. arousal & $3.94(1.47)$ & $1.56(1.37)$ & 0.001 \\
& Severity re-experiencing & $5.61(4.25)$ & $2.00(2.99)$ & 0.001 \\
& Severity avoidance & $12.22(4.82)$ & $3.25(4.11)$ & 0.001 \\
& Severity arousal & $9.28(4.06)$ & $2.63(2.85)$ & 0.001 \\
Current trauma & Intrusion & $20.50(7.10)$ & $4.19(5.62)$ & 0.001 \\
symptoms IES & Avoidance & $21.33(10.83)$ & $3.44(4.79)$ & 0.001 \\
DSM-IV PTSD & $\%$ meeting criteria & 78 & 25 & 0.001 \\
& & & & \\
\hline
\end{tabular}

PDS, Post-traumatic Diagnostic Scale; IES, Impact of Events Scale; PTSD,

post-traumatic stress disorder.

Values in parentheses are standard deviations.

${ }^{a}$ Data only from those participants who report traumatic life events.

Together, the trauma variables were found to significantly predict beliefs regarding malevolence $[F(3,30)$ $=22.77, p<0.001]$, benevolence $[F(3,30)=11.37, p<$ $0.001]$ and omnipotence $[F(3,30)=9.12, p<0.001]$, accounting for $66.4,48.5$ and $42.5 \%$ of variance in beliefs respectively. For each analysis, standardized regression coefficients indicated that the total score on the IES was the only significant predictor in the equation for malevolence $(\beta=0.91, t=6.98, p<0.001)$, benevolence $(\beta=-0.25, t=-4.95, p<0.001)$ and omnipotence $(\beta=0.19, t=4.23, p<0.001)$. Thus, these results clearly show that current symptoms related to past traumatic events are significant predictors of a person's beliefs about their voices, with greater current trauma symptoms being associated with more malevolence and omnipotence (and less benevolence) of the voice.

Regression analyses were also conducted to determine the way in which trauma and beliefs were related to distress. Separate analyses were conducted for each of the dependent variables: distress as measured by the BDI-II and by the BAI. The following independent variables were included in the regression analysis: number of traumatic events, IES total score, beliefs about malevolence, frequency of voices, and the amount of negative voice content. Together these variables accounted for a significant proportion $(58.7 \%)$ of the variance in the dependent variable of distress as measured by the BDI-II $[F(5,37)=10.38$, $p<0.001]$. Regression coefficients indicated that the only significant predictor of distress as measured by the BDI-II was beliefs about malevolence $(\beta=1.30$, $t=2.34, p<0.05)$. The variables also accounted for a significant proportion $(62.2 \%)$ of the variance in the independent variable of distress as measured by the BAI $[F(5,37)=11.88, p<0.001]$. Standardized regression coefficients indicated that the only significant predictor of distress as measured by the BAI was the total IES score $(\beta=0.28, t=2.39, p<0.05)$.

\section{Discussion}

This study aimed to explore the relationship between trauma and voices specifically in the context of a cognitive-behavioural model of voices. In doing so, we detected a high prevalence of traumatic life events among both PVH and NPVH, but a significantly higher prevalence of persistent psychological symptoms consistent with PTSD among PVH. Specific trauma variables accounted for a significant proportion of the variance on three of the belief about voices subscales: malevolence, benevolence and omnipotence. The only significant predictor in all of these regression equations was the total symptom score on the IES. These results suggest not only that a number of trauma variables play a role in the interpretation of voices but also that a particularly important factor in determining beliefs about voices might be whether the psychological effects of the trauma persist (that is the extent to which the trauma has been resolved).

Further analyses were carried out to explore the extent to which the number of traumatic events, the degree of unresolved trauma, beliefs about malevolence and key voice characteristics contributed to distress. In terms of depression, these factors were found to account for a significant proportion of the variance. However, the only significant predictor of distress was beliefs about malevolence. This suggests that although trauma, voice content and voice frequency may contribute towards depression, beliefs about voice malevolence are a superior predictor of depression. With regard to anxiety, the same variables also accounted for a significant proportion of the 
variance, but the only significant predictor was the total score on the IES. This finding is somewhat intuitive as the symptoms found on the IES (intrusions and avoidance) are typical of an anxiety disorder and would therefore be highly likely to predict anxiety. However, together with the above finding there are a number of important implications. It would appear that, in terms of distress, it is important to consider both trauma factors and voice factors as the levels of heightened anxiety observed in PVH that have previously been attributed to the beliefs about voices may be better accounted for by trauma symptoms.

This study detected a high prevalence of trauma in both voice-hearing groups; a level much higher than the general population (Kessler et al. 1995). These findings are consistent with previous studies of psychiatric populations that have estimated that 70-98\% of this population report exposure to trauma (e.g. Mueser et al. 1998; Frame \& Morrison, 2001; Neria et al. 2002). Few studies have directly compared the traumatic life experiences of psychiatric- and nonpsychiatric voice hearers. Honig et al. (1998) detected significant levels of trauma in both a psychiatric and non-psychiatric group of voice hearers, although the authors focused almost exclusively on child abuse in their analysis and discussion of the results. Romme \& Escher (1989) also discuss a high prevalence of trauma in the two groups included in their studies ('copers' and 'non-copers'). The authors reported that $70 \%$ of the sample felt that their voices had been triggered either directly by a traumatic life event or by a reactivation of past trauma. However, the definition of trauma was somewhat broad and subjective and included events that elicited an intense emotional response such as being in love, being pregnant, or illness. The present study used stricter psychiatric diagnostic criteria for the definition of a traumatic event and still detected a high prevalence of trauma. The data collected here permitted a direct comparison of the types of traumatic events experienced by the two groups. Both groups reported experiencing a variety of traumatic life events in both childhood and adulthood, but significantly more individuals in the $\mathrm{PVH}$ group reported experiencing childhood sexual abuse. This is consistent with previous findings with groups of individuals with psychosis (e.g. Goff et al. 1991) and specifically with voice hearers (Honig et al. 1998; Read et al. 2003). Abuse in childhood is clearly an important factor in the genesis of psychotic symptoms and the mediation of distress. It has been shown to be a significantly greater predictor of voice characteristics than abuse in adulthood (Read et al. 2003). Moreover, individuals with a history of abuse were found by Read et al. (2003) to be significantly more likely to experience command hallucinations to harm or kill themselves than those who do not report abuse.

The results from the current study suggest that, in individuals with trauma histories, beliefs about voices may be at least partially understood in the context of trauma, that is the nature of the trauma, the meaning of the trauma to the individual, and the extent to which the trauma has been resolved. This adds further weight to the emerging argument (see Larkin \& Morrison, 2006) for a formulation-based approach to psychosis that would integrate, where appropriate, trauma potentially either as a vulnerability, precipitating or maintaining factor in the distress associated with psychosis. For example, an individual who has been repeatedly sexually assaulted may feel that they have no control over the actions of others, or that others cannot be trusted and are 'out to get them' (see Morrison, 2001). It is of little surprise, then, that PVH interpret their voices as significantly more malevolent and omnipotent, and experience significantly more severe depression and anxiety in association with the voices.

On a theoretical level, the detection here of a high prevalence of traumatic life events among $\mathrm{PVH}$ and NPVH lends support to recent psychological models of psychotic symptoms (Garety et al. 2001; Morrison, 2001). Our findings suggest that traumatic life events could act as a vulnerability or precipitating factor for the development of psychotic symptoms (hence the similar levels of life events in each group) but that the nature of the trauma and the extent to which it remains unresolved may represent a maintaining factor by colouring the individual's beliefs about the voices they hear.

The findings from this study raise a number of clinical implications. The high prevalence of childhood sexual abuse within the PVH group, although not of statistical significance as a predictor of beliefs about voices, remains an important finding of the present study. It was the only trauma that significantly more PVH had reported than NPVH. Previous research suggests that individuals with a diagnosis of 'schizophrenia' are less likely to be asked about abuse (Read \& Fraser, 1998a) or to receive an adequate response when abuse is disclosed (Agar \& Read, 2002). Indeed, in one study it was shown that very few individuals with a diagnosis of 'schizophrenia' were referred for abuse counselling (Read \& Fraser, 1998b). The high prevalence of childhood sexual abuse in PVH suggests that this may be an important factor to consider in the assessment, formulation and intervention of individuals who hear voices. This is unlikely to occur in the absence of staff training (Read et al. 2007).

This study included an interesting sample of NPVH in an attempt to join others (see Morrison, 2001) in 
elucidating the way in which trauma may be related to the development and maintenance of distressing psychotic symptoms. There were, however, several methodological limitations. Unfortunately, the sample in this study is very small because it was not funded and was conducted over a very short period of time. Any interpretation of our findings must take this into consideration. Even though our results cannot, in isolation, be generalized, they are consistent with the emerging literature on trauma and psychosis, and trauma and voices in particular. Among this developing literature there seems to be little agreement on which tool is the most appropriate for assessing trauma in this population. Whereas some have used a modified version of the Trauma History Questionnaire, others have used an interview approach to establish the incidence of trauma in an individual's past. We chose the PDS as a self-report measure in the hope that being asked to just tick a box indicating the occurrence of a specific form of trauma would maximize disclosure. We also felt that it was unethical to ask detailed questions about trauma outside of a trusting, therapeutic relationship. The PDS has not, however, been standardized for use in this population $(\mathrm{PVH})$, and there is some concern that what the symptom measures are in fact detecting are the symptoms of psychosis rather than pure trauma per se (see Morrison, 2001). This will always be a thorny issue when assessing trauma symptoms in this population but it does mean that the 'diagnosis' of PTSD in this study needs to be interpreted with caution.

It must be considered whether the non-psychiatric population here is representative of voice hearers in the general population. Previous studies (e.g. Romme \& Escher, 1989) have excluded mediums from voice-hearing studies as it was speculated that they experience 'pseudo-hallucinations' and thus their experiences are not directly comparable to those of a psychiatric population. However, we found that those in the NPVH group were able to rate their voices and experiences in much the same way as the $\mathrm{PVH}$ group and both groups described hearing voices originating from both inside and outside of their head. It was therefore felt that they were a valid voice-hearing group. It may be, however, that had we had funding to advertise in the media for voice hearers (as Romme \& Escher did in their seminal study), we may have found a broader, more representative population of NPVH. We did not screen our non-psychiatric population for any form of diagnosable mental health problem. Given that none had been involved with psychiatric services, it could be assumed that they did not have a major mental illness but this was not assessed formally. Nonetheless, they were not distressed by their experiences and they did not hold negative beliefs about their hallucinatory experiences. Objective data (i.e. obtained by structured clinical interview) on diagnosis were not collected from the psychiatric population. Although based on self-reports, all PVH had a diagnosis of a schizophreniform disorder, and it would have been more valid to have used a structured clinical interview to ascertain diagnosis.

Despite these limitations, it remains likely that trauma and symptoms (including hearing voices) are related in complex and reciprocal ways. This study and the emerging body of research that inspired it emphasize the need to consider the role of trauma in the development and maintenance of voice hearing and other serious mental health problems.

\section{Acknowledgements}

Dr Andrew gratefully acknowledges the South Wales Training Course in Clinical Psychology.

\section{Declaration of Interest}

None.

\section{References}

Agar K, Read J (2002). What happens when people disclose sexual or physical abuse to staff in a community mental health centre? International Journal of Mental Health Nursing 11, 70-79.

APA (1994). The Diagnostic and Statistical Manual of Mental Disorders, 4th edn. American Psychiatric Association: Washington, DC.

Bebbington P, Bhugra D, Bhugra T, Singleton N, Farrell M, Jenkins R, Lewis G, Meltzer H (2004). Psychosis, victimization and childhood disadvantage: evidence from the second British National Survey of Psychiatric Morbidity. British Journal of Psychiatry 185, 220-226.

Beck AT, Epstein N, Brown G, Steer RA (1988). An inventory for measuring clinical anxiety: psychometric properties. Journal of Consulting and Clinical Psychology 56, 893-897.

Beck AT, Steer RA, Brown GK (1996). Manual for Beck Depression Inventory - II. Psychological Corporation: San Antonio, TX.

Bentall RP (1990). The illusion of reality: a review and integration of psychological research on hallucinations. Psychological Bulletin 107, 82-95.

Birchwood M, Meaden A, Trower P, Gilbert P, Plaistow J (2000). The power and omnipotence of voices: subordination and entrapment by voices and significant others. Psychological Medicine 30, 337-344.

Bryman A, Carmer D (1997). Quantitative Data Analysis with SPSS for Windows: A Guide for Social Scientists. Routledge: London.

Chadwick PD, Birchwood MJ (1994). The omnipotence of voices: a cognitive approach to hallucinations. British Journal of Psychiatry 164, 190-201. 
Chadwick P, Lees S, Birchwood M (2000). The revised Beliefs About Voices Questionnaire (BAVQ-R). British Journal of Psychiatry 177, 229-232.

Close H, Garety P (1998). Cognitive assessment of voices: further developments in understanding the emotional impact of voices. British Journal of Clinical Psychology 37, 173-188.

Day S, Peters E (1999). The incidence of schizotypy in new religious movements. Personality and Individual Differences 27, 101-116.

Ensink BJ (1992). Confusing Realities. A Study on Child Sexual Abuse and Psychiatric Symptoms. Free University Press: Amsterdam.

Falmularo R, Kinscherff R, Fenton T (1992). Psychiatric diagnoses of maltreated children: preliminary findings. Journal of the American Academy of Child and Adolescent Psychiatry 31, 863-867.

Foa EB (1995). Posttraumatic Stress Diagnostic Scale. National Computer Systems: Minneapolis, MN.

Frame L, Morrison A (2001). Causes of posttraumatic stress disorder in psychotic patients. Archives of General Psychiatry 58, 305-306.

Garety PA, Kuipers E, Fowler D, Freeman D, Bebbington PE (2001). A cognitive model of the positive symptoms of psychosis. Psychological Medicine 31, 189-195.

Goff DC, Brotman AW, Kindlon D, Waites M, Amico E (1991). Self-reports of childhood abuse in chronically psychotic patients. Psychiatry Research 37, 73-80.

Haddock G, MaCarron J, Tarrier N, Faragher E (1999). Scales to measure dimensions of hallucinations and delusions: the psychotic symptoms rating scales (PSYRATS). Psychological Medicine 29, 879-889.

Honig A, Romme M, Ensink B, Escher S, Pennings M, Devries M (1998). Auditory hallucinations: a comparison between patients and nonpatients. Journal of Nervous and Mental Disease 186, 646-651.

Horowitz M, Wilner N, Alvarez W (1979). Impact of Event Scale: a measure of subjective distress. Psychosomatic Medicine 41, 209-218.

Kessler R, Sonnega A, Bromet E, Hughes M, Nelson B (1995). Posttraumatic stress disorder in the National Comorbidity Survey. Archives of General Psychiatry 52, 1048-1060.

Kilcommons A, Morrison AP (2005). Relationships between trauma and psychosis: an exploration of cognitive and dissociation factors. Acta Psychiatrica Scandinavica 112, 351-359.

Larkin W, Morrison A (eds) (2006). Trauma and Psychosis. Routledge: London.

Lothian J, Read J (2002). Asking about abuse during mental health assessments: clients' views and experiences. New Zealand Journal of Psychology 31, 98-103.
McGreery CA, Claridge GA (1995). Out-of-body experiences and personality. Journal of the Society for Psychical Research 60, 129-148.

Morrison A (2001). The interpretation of intrusions in psychosis: an integrative cognitive approach to hallucinations and delusions. Behavioural and Cognitive Psychotherapy 29, 257-276.

Mueser KT, Goodman LB, Trumbetta SL, Rosenberg SD, Osher FC, Vidaver R, Aucellio P, Foy DW (1998). Trauma and posttraumatic stress disorder in severe mental illness. Journal of Consulting and Clinical Psychology 66, 493-499.

Neria Y, Bromet E, Sievers S, Lavelle J, Fochtman L (2002). Trauma exposure and post-traumatic stress disorder in psychosis: findings from a first admission cohort. Journal of Consulting and Clinical Psychology 70, 246-251.

Raune D, Kuipers E, Bebbington P (1999). Psychosocial stress and delusional verbal auditory hallucinatory themes in first episode psychosis: implications for early intervention. Paper presented at Psychological Treatments of Schizophrenia, Oxford, 23-24 September.

Read J, Agar K, Argyle N, Aderhold V (2003). Sexual and physical abuse during childhood and adulthood as predictors of hallucinations, delusions and thought disorder. Psychology and Psychotherapy: Theory, Research, Practice 76, 1-23.

Read J, Fraser A (1998a). Abuse histories of psychiatric inpatients: to ask or not to ask? Psychiatric Services 49, 355-359.

Read J, Fraser A (1998b). Staff response to abuse histories of psychiatric inpatients. Australian and New Zealand Journal of Psychiatry 32, 206-213.

Read J, Hammersely P, Rudegeair T (2007). Why, when and how to ask about childhood abuse. Advances in Psychiatric Treatment 13, 101-110.

Romme M, Escher A (eds) (1993). Accepting Voices. Mind: London.

Romme MAJ, Escher A (1989). Hearing voices. Schizophrenia Bulletin 15, 209-216.

Ross C, Joshi S (1992). Schneiderian symptoms and childhood trauma in the general population. Comprehensive Psychiatry 33, 269-273.

Thalbourne MA (1994). Belief in the paranormal and its relationship to schizophrenia-relevant measures: a confirmatory study. British Journal of Clinical Psychology 33, 78-80.

Tobert N (2001). The polarities of consciousness. In Psychosis and Spirituality: Exploring the New Frontier (ed. I. Clarke), pp. 29-52. Whurr Publishers: London.

Young M, Read J, Barker-Collo S, Harrison R (2001). Evaluating and overcoming barriers to taking abuse histories. Professional Psychology: Research and Practice 32, 407-414. 